\title{
Chronic pancreatitis as a cause of gastrointestinal bleeding
}

\author{
R I HALL, M I LAVELLE, AND C W VENABLES \\ From The Professorial Surgical Unit and Department of Radiology, Royal Victoria Infirmary, Newcastle \\ upon Tyne
}

SUMMARY Chronic pancreatitis is an infrequently considered cause of gastrointestinal bleeding. Four cases are described who presented to a surgical unit in a year. One patient bled down the main pancreatic duct from a splenic artery pseudoaneurysm, one had a fatal haemorrhage from a superior mesenteric artery aneurysm which ruptured into the duodenum, and two were considered to have bled from vessels in the stomach or colon which were involved in the peripancreatic inflammatory tissues. The difficulties in diagnosing these patients are described and the report emphasises that the diagnosis should be considered in obscure cases of gastrointestinal bleeding especially where there is a history of alcohol abuse and left upper quadrant or epigastric pain.

In the United Kingdom chronic pancreatitis is rarely considered in the diagnosis of gastrointestinal bleeding and often does not feature in reviews of the subject. ${ }^{1}$ Our own experience and a review of the literature suggest that it may be a more frequent cause than is realised, a recent report being able to describe 12 cases of bleeding at one institution due to this cause, and to cite another 60 in the recent literature. ${ }^{2}$

We report four cases who presented to a surgical unit in one year, three of whom had multiple investigations for their recurrent gastrointestinal bleeding, and were eventually found to have bled as a result of their chronic pancreatitis.

\section{Case 1}

JD, a 50 year old man, had been previously fit and admitted to only moderate drinking. He complained of intermittent left upper quadrant pain radiating to his back for one year. Six months after the pain began he had a large haematemesis for which a laparotomy was performed at his local hospital and, though no cause for the bleeding was found, the pancreas was noted to be nodular and sclerosed. He subsequently had several more haematemeses associated with left upper quadrant pain and gastroduodenoscopy on two occasions failed to show any lesion to account for the bleeding.

He was referred for endoscopic retrograde cholangiopancreatography (ERCP) but on the day before admission he had further pain and a large haematemesis causing his haemaglobin to fall to $5.6 \mathrm{~g} / \mathrm{dl}$.

Received for publication 18 August 1981
Abdominal examination revealed a tender fullness in the right hypochondrium and it was noted that his serum amylase was raised to $1950 \mathrm{IU} / 1$.

ERCP was performed, and revealed a cyst two-thirds of the way along the main pancreatic duct which filled with dye and then rapidly emptied. In view of the known risks of injecting dye into pancreatic cysts at ERCP the patient underwent laparotomy the same day. At operation changes of chronic pancreatitis were found and the cyst was found to be a pseudoaneurysm of the splenic artery in communication with the main pancreatic duct. This was presumed to be the route of bleeding. A distal pancreatectomy was performed, resecting the aneurysm along with the tail of the pancreas and the spleen.

Postoperatively the patient made a smooth recovery, has had no more bleeds, and maintains a normal serum amylase.

\section{Case 2}

AF, a 57 year old male, had a six year history of abdominal pain secondary to alcoholic chronic pancreatitis which had been confirmed by ERCP and coeliac axis angiography in 1976.

In 1979 the patient complained of increasing pain, weight loss, and steatorrhoea. An ultrasound scan showed the presence of a $5 \mathrm{~cm}$ cyst in the head of the pancreas and a repeat scan six months later showed the development of a second cyst in the neck of the gland and dilatation of the biliary tree. At this time the patient's haemoglobin was 10.5 , amylase was $835 \mathrm{IU} / 1$, while alkaline phosphatase was $158 \mathrm{IU} / \mathrm{l}$ with a normal bilirubin. 


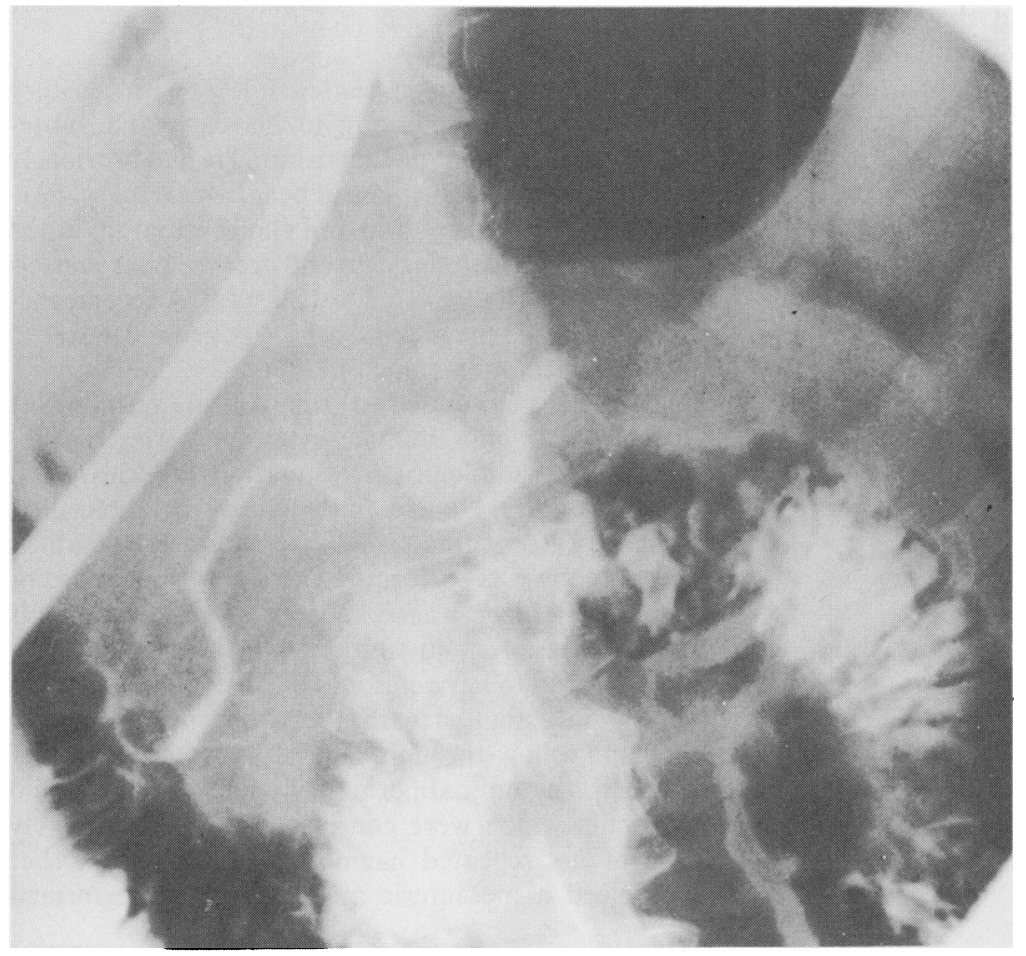

Fig. 1 Case 1. A large cyst is visualised two-thirds of the way along the main pancreatic duct.

Fig. 2 Case 1. The resected specimen. The probe enters the splenic artery which is clearly shown opening into the cyst on the main pancreatic duct. 
Laparotomy was performed and the diagnosis of chronic pancreatitis confirmed. Inflammatory tissue was found to be causing partial pyloric obstruction and marked obstruction of the common bile duct despite his unremarkable liver function tests. One of the cystic lesions was shown by operative pancreatogram to be connected to the main pancreatic duct but the second was a large pseudo-aneurysm of the superior mesenteric artery.

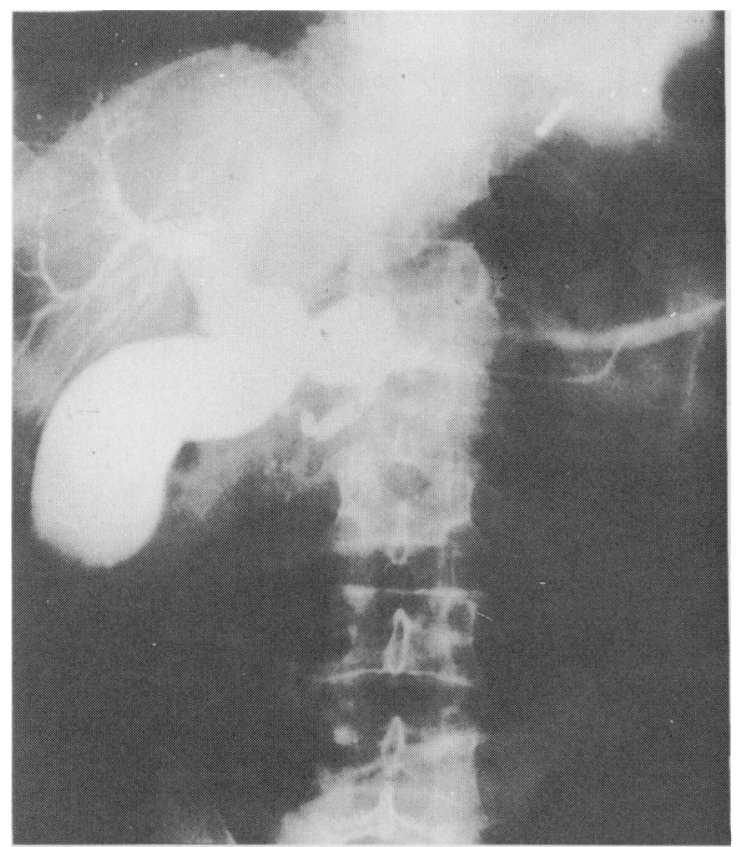

Fig. 3 Case 2. Peroperative cholangiogram and tube pancreatograms showing gross biliary obstruction and $a$ cyst connected to the middle of the main pancreatic duct.

A distal pancreatectomy with splenectomy, choledochoduodenostomy, gastroenterostomy, and cystdrainage was performed. The aneurysm was considered too hazardous to resect and was, therefore, left in situ.

Postoperatively the patient was considerably improved and his amylase and alkaline phosphatase returned to normal. Two months later, however, he had another attack of pain and a massive haematemesis. Gastroscopy showed the stomach to be completely full of blood and, despite an immediate laporotomy, he died.

The cause of the bleeding was found to be a fistula between the superior mesenteric artery and the duodenum.
Case 3

RS, a 57 year old man, presented in 1977 with a history of epigastric pain radiating to his back and subsequently into the left hypochondrium. He had previously been well and drank six pints of beer twice weekly. Barium meal, gastroscopy, and oral cholecystogram failed to show any cause for his pain and over the next year his haemoglobin fell from $16 \cdot 2$ to $12 \cdot 2 \mathrm{~g} / \mathrm{dl}$. A second gastroscopy showed the presence of coffee ground material in the stomach but no mucosal lesion.

The patient continued to complain of constant left hypochondrial pain but an extensive series of biochemical and radiological investigations failed to reveal any abnormality. He had an episode of rectal bleeding and shortly afterwards a small haematemesis for which a gastroscopy was performed and a very small cardiooesophageal ulcer was diagnosed. At this time his serum amylase was noted to be $479 \mathrm{IU} / 1$. One month later he began to have episodes of fresh rectal bleeding and was investigated with a barium enema, barium meal, and follow-through, another gastroscopy, and a ${ }^{99} \mathrm{Tc}$ scan, the only abnormality being a few sigmoid diverticula which were considered to be an unlikely cause of his repeated haemorrhages. After another large bleed a mesenteric angiogram was performed

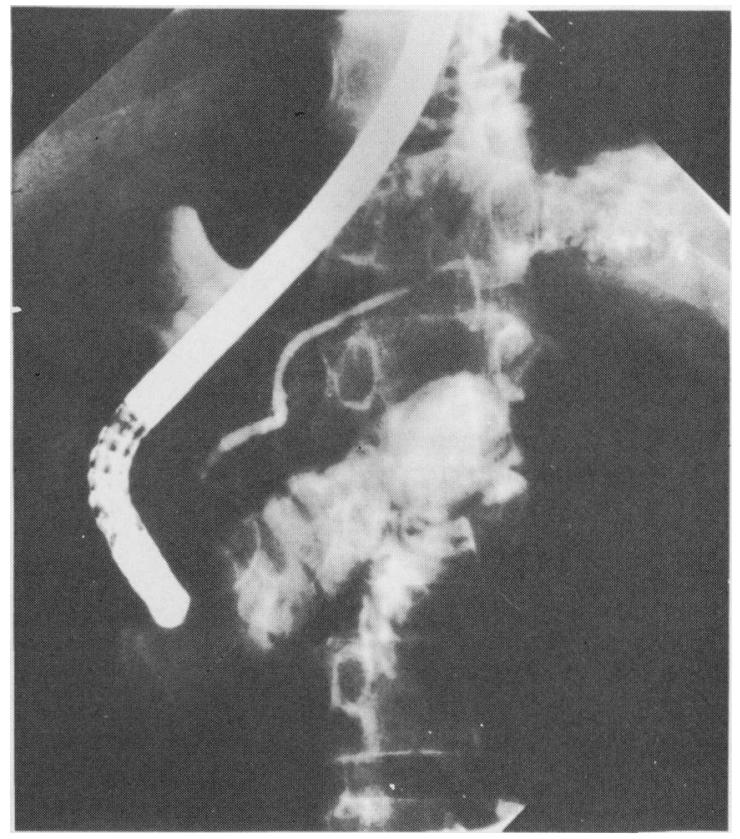

Fig. 4 Case 3. The distal third of the main duct shows strictures, irregular dilatation, and intraduct calcification. 
which showed some pooling of contrast in the left flank, thought to be in the jejunum, but no clear vascular abnormality.

Prompted by the single finding of a raised amylase a pancreatic isotope scan was performed and this showed poor uptake in the distal pancreas. ERCP was performed and this revealed a stricture of the main duct with calcification and changes typical of chronic pancreatitis.

The patient subsequently underwent laparotomy at which the pancreas was found to be grossly inflamed and dense vascular adhesions were found to involve the stomach and transverse colon, though it was not possible to identify any mucosal breach in either organ. The spleen was of normal size.

After distal pancreatectomy and splenectomy the patient made a smooth recovery and to date has had no further bleeds.

\section{Case 4}

$\mathrm{JN}$, a 39 year old male, presented in 1977 with an attack of acute pancreatitis presumed to be secondary to chronic alcoholism. In 1980 he had an episode of left upper quadrant pain and a large haematemesis, at which time his serum amylase was normal and gastroscopy showed altered blood in the stomach but no mucosal lesion. Shortly afterwards he was readmitted with another bleed and endoscopy again revealed only altered blood. One month later he was readmitted to investigate a falling haemoglobin and was noted to have melaena stools. A third gastroscopy was essentially normal, as were barium meal and follow-through examinations.

During this admission a slightly raised serum amylase of $356 \mathrm{UL} / 1$ was noted and a pancreatic aetiology for his bleeding was considered. Coeliac axis angiography revealed increased vascularity of the tail of the gland and ERCP showed irregularity and dilatation of the main duct with bleeding and cystic changes of the side radicles, indicative of chronic pancreatitis.

These findings made it very likely that chronic pancreatitis was the cause of the bleeding and the patient agreed to laparotomy. At operation the whole distal pancreas was grossly inflamed and a small pancreatic cyst, surrounded by large veins, was found densely adherent to the posterior wall of the stomach. The spleen was of normal size and after a distal pancreatectomy and splenectomy the patient made an uneventful recovery with no more bleeds at the time of writing.

\section{Discussion}

Chronic pancreatitis may cause gastrointestinal bleeding either via the main pancreatic duct or from eroded vessels in the walls of neighbouring viscera. ${ }^{3}$ The former route has been termed haemosuccus pancreaticus ${ }^{4}$ or Wirsungorrhagia ${ }^{6}{ }^{7}$ and has been described on several occasions, ${ }^{89}$ although few of these cases describe splenic artery aneurysms actually communicating with the duct as in case 1 . Visceral vessel aneurysms are surprisingly common in chronic pancreatitis ${ }^{5}{ }^{10}$ and they are usually found on the splenic vessels, sometimes on the

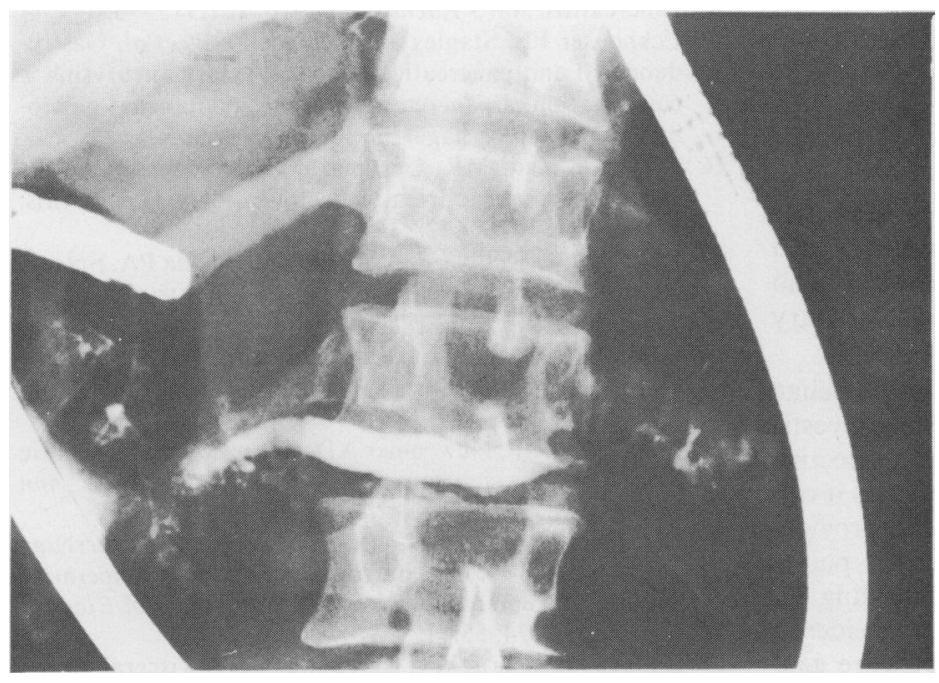

Fig. 5 Case 4. The distal main duct shows cystic dilatations and beading with similar changes in the side radicles. 
pancreaticoduodenal arcade, ${ }^{11}$ and only rarely do they involve the superior mesenteric artery as in case $2 .{ }^{12}$

The other two patients appear to have bled from eroded vessels in the stomach or colon, although no precise source of blood loss was demonstrated. The presence of vascular adhesions and dilated veins raises the possibility of segmental portal hypertension due to splenic vein thrombosis, which can occur in chronic pancreatitis. This may cause bleeding from varices in the stomach ${ }^{13}$ or colon, ${ }^{14}$ but our patients did not have obvious splenic vein thrombosis, nor did they have splenomegaly, which is nearly always its sequel in chronic pancreatitis. ${ }^{15}$

The identification of a probable pancreatic aetiology for the three causes of recurrent bleeding described here was not difficult once the possibility had been considered. Before this all three had numerous endoscopic and barium examinations to no avail, confirming Starling's opinion that such investigations are time-consuming and ineffective in chronic pancreatitis. ${ }^{5}$ Certain clinical features may suggest the pancreas to be the source of the bleeding and a triad of left upper quadrant pain, recurrent gastrointestinal bleeds, and a history of chronic pancreatitis has been described in patients bleeding down the main duct.' A history of alcohol abuse or a previous attack of acute pancreatitis should also cause the pancreas to be suspected.

The most appropriate investigation to confirm the diagnosis in most reports is said to be coeliac axis

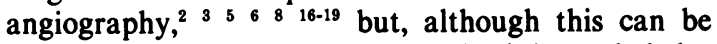
arranged in an emergency, in practice it is rarely helpful and in one patient in whom it was performed during a bleed it failed either to localise the bleeding point or to demonstrate chronic pancreatitis. In two other cases angiography showed changes consistent with chronic pancreatitis.

Ultrasonic scan was helpful in one case where it showed a diseased pancreas and biliary obstruction, but it failed to distinguish between a cyst and an aneurysm, a problem which has been noted previously. ${ }^{12}$

In our experience the most helpful investigation has been ERCP, which demonstrated the bleeding point in case 1 and revealed a diseased pancreas in the other patients. Successful results from ERCP have also been reported by Bowes, ${ }^{20}$ Rosch, ${ }^{21}$ and by Fraissinet ${ }^{7}$ who used ERCP and angiography as complementary investigations.

In the acutely bleeding patient, therefore, coeliac axis angiography may be the most appropriate investigation if only to exclude other causes, but, as an elective procedure, ERCP appears to be more helpful, as it can readily demonstrate chronic pancreatitis and provide, at the very least, circumstancial evidence that points towards the pancreas as the cause of the bleeding. In our own opinion this investigation should be considered whenever patients are encountered with obscure gas- trointestinal bleeding, particularly where there is a history of alchohol abuse, epigastric or left upper quadrant pain, or previous pancreatic disease. In such cases the pancreas should be excluded as a cause of bleeding rather than subject the patient to repeated negative endoscopies and contrast studies.

\section{References}

1 Thomas G. The clinical presentation of acute upper gastrointestinal bleeding. Br J Hosp Med 1980; 23/4:333-7.

2 Stanley JC, Frey CF, Miller TA, Lindenauer SM, Child CG, III. Major arterial haemorrhage, a complication of pancreatic pseudo cysts and chronic pancreatitis. Arch Surg 1976; III:435-40.

3 Scandblom P. Gastrointestinal haemorrhage through the pancreatic duct. Ann Surg 1970; 171:61-6.

4 Bivins BA, Sachatello CR, Chuang VP, Brady P. Haemosuccus pancreaticus; gastrointestinal haemorrhage due to rupture of a splenic artery aneurysm into the pancreatic duct. Arch Surg 1978; 113:751-3.

5 Starling JR, Crummy AB. Haemosuccus pancreaticus secondary ruptured splenic artery aneurysm. Dig Dis Sci 1979; 9:726-9.

6 Favriel JM, Cerf M, Sterin P, et al. Rupture d'une aneurysm de l'artere splenique dans la pancreas, cause exceptionelle d'haemorrhagie digestive par Wirsungorrhagia. SEM HOP (Paris) 1979; 55:1125-8.

7 Fraissinet R, Sahal J, Sarles H. Wirsungorrhagia. Report of a case and review of the literature. Gastroenterol Clin Biol 1978; 2:99-100.

8 Corbeau A, Sahal J, Fraissinet R, Cocjaret P, Burelle H, Clement JP. Haemorrhage from the duct of Wirsung: emergency arteriographic diagnosis. One case. $J$ Radiol Electrol Med Nur 1918; 59:275-8.

9 Kelch L. Gastrointestinal haemorrhage into the duct of Wirsung caused by chronic pancreatitis and carcinoma of the pancreas. Med Klin 1974; 25:1008-11.

10 White AF, Baum S, Buronasin S. Aneurysm secondary to pancreatitis. Am J Roentgenol 1976; 127:373-96.

11 Eckhouser FE, Stanley JC, Zelenoch GB, et al. Gastroduodenal and pancreatico-duodenal artery aneurysms: a complication of pancreatitis causing spontaneous gastrointestinal haemorrhage. Surgery 1980; 9:335-44.

12 Kouae A, Zadi MR, Geshner J. False aneurysm of the superior mesenteric artery; a complication of pancreatitis. Br J Radiol 1979; 622:836-8.

13 Khan CH, O'Reilly CJ, Avakain VA, Lucina PA. Splenic vein thrombosis: an unusual cause of gastric bleeding. Angiology 1977; 28:725-7.

14 Burbige EJ, Tarder G, Casia S, Eugene J, Frey C. Colonic varices, a complication of pancreatitis with splenic vein thrombosis. Am J Dig Dis 1978; 23:252-5.

15 Longstrech GF, Newcomer AD, Green PA. Extrahepatic portal hypertension caused by chronic pancreatitis. Ann Intern Med 1971; 75:903-7.

16 Chermet J, Bigot JM, Monier JP. Digestive haemorrhage due to arterial erosions during pancreatitis. Pre-operative diagnosis by emergency arteriography. $J$ Radiol Electrol Med Nucl 1974; 55:117-22.

17 Gadacz TR, Trunkey D, Keiffer RF. Visceral vessel 
erosions associated with pancreatitis, case reports and review of the literature. Arch Surg 1978; 113:1438-40.

18 Lam AY, Brichess RS. Pancreatic pseudo cyst with haemorrhage into the gastrointestinal tract through the duct of Wirsung. Am J Surg 1975; 129:694-95.

19 Soeno T, Kakizaki G. Massive haemorrhage into the upper digestive tract due to rupture of splenic artery aneurysm into the pancreas. Am J Gastroenterol 1974; 61:55-62.
20 Bowers J, Koehler PR, Hammar SP, Nelson JA, Tolman KG. Rupture of splenic artery aneurysm into the pancreatic duct. Gastroenterology 1976; 70:1152-5.

21 Rosch W, Shaffner O, Fruhmorgen P, Koch H. Massive gastrointestinal haemorrhage into the pancreatic duct. Diagnosed by duodenoscopy and ERCP. Endoscopy 1976; 8:93-6. 